\title{
FRACTURE RESISTANCE OF MOLAR TEETH RESTORED BY ENDOCROWN AND ONLAY CAD/CAM MONOLITHIC CERAMIC MATERIALS. AN IN-VITRO STUDY
}

\author{
Mohamed S.M Abdelrahman*, Hesham I Othman**, Mohamed F Metwally***
}

\begin{abstract}
Objective: This study evaluated the Fracture Resistance of Molar Teeth Restored by Endocrown and Onlay CAD/CAM Monolithic Ceramic Materials.

Material and Methods: twenty-four human mandibular molar teeth were used in this study. A total of twenty-Four samples were randomly divided into two main groups (12 each). Group A: teeth were prepared and restored by endocrown restorations. Group B: teeth were prepared and restored by onlay restorations. Each group was divided into two subgroups; Subgroup 1: restorations were fabricated from IPS E. Max Cad, and subgroup 2: restorations were fabricated from Vita Enamic. The restorations were cemented using a dual-cure resin cement, then specimens were subjected to fracture resistance test.
\end{abstract}

Results: Two-way ANOVA test, post hoc Tuckey test and descriptive statistics were used to compare different designs and materials. The significance level was set to $\mathrm{P} \leq 0,05$. Intergroup comparisons while controlling the materials revealed that with Emax, there was no statistically significant difference between endocrown and onlay $(\mathrm{P}=0.3)$. As for Enamic, there was no statistically significant difference between endocrown and onlay $(\mathrm{P}=0.95)$. When the type of restoration was controlled to compare different materials, there was a statistically significant difference between Emax and Enamic endocrowns ( $\mathrm{P}=0.02)$. As for Onlay restorations, there was no statistically significant difference between Emax and Enamic $(\mathrm{P}=0.11)$.

Conclusion: Within the limits of this in vitro study, the following can be concluded: Endocrowns and onlays can be used safely in terms of fracture resistance as both have values which exceed the physiologic requirements..

KEYWORDS: CAD/CAM, Endocrown, Onlay, Endodontically Treated Molars.

\section{INTRODUCTION}

The restoration of endodontically treated teeth (ETT) has been a controversial topic for many years. It is known that vitality loss causes physical and structural changes affecting the dentin properties such as micro-hardness, modulus of elasticity and fracture toughness. In order to restore the endodontically treated teeth, often a special approach is required, due to the considerable loss of tooth structure, a preexisting carious or non-carious coronal lesions, a previous restoration and the endodontic treatment itself. On the other hand, the diminished resistance to functional occlusal forces of the remaining hard dental tissues is an obvious clinical observation. Some aspects concerning the etiology of this situation are still controversial. The change in tooth moisture content due to loss of vitality has a slight influence on Young's modulus ${ }^{(1)}$. This change in water content has no influence in decreasing compressive and tensile strength ${ }^{(1)}$.

* B.D.S 2012 G.,Faculty of Dental Medicine, Boys, Cairo, Al-Azhar University .Dentist at Ministry of health, Al-Dakahlia-BanyAbid

** Professor of Crown and bridge, Faculty of Dental Medicine for Boys, Al-Azhar University.

*** Lecturer of Crown and bridge, Faculty of Dental Medicine for Boys, Al-Azhar University. 
The specific biomechanical behavior of endodontically treated teeth is first induced by the loss of hard dental tissues because of carious and non-carious lesions, which may lead to a preexistent cavity, even before the endodontic therapy is initiated, which adds to the loss of tooth structure induced by the endodontic access cavity preparation. Removing the pulp chamber roof, with consequent deepening of the whole cavity will increase flexure possibilities of the vertical coronal walls, which become more prone to fracture ${ }^{(2)}$. A conservative endodontic access cavity preparation will affect tooth stiffness with only $5 \%$. The subsequent root canal therapy (instrumentation and obturation) will lead to a slight reduction in fracture resistance ${ }^{(3)}$ or will have little or no effect on biomechanical properties of the tooth ${ }^{(4)}$. Cleaning and shaping the root canal system diminishes tooth stiffness in proportion to the amount of removed tissues, and it is possibly related to the chemical or structural alteration brought about by endodontic chemical products $^{(5)}$.

The amount of remaining tooth structure is probably the single most important predictor of clinical success ${ }^{(6)}$. In most cases the remaining tooth structure is limited as a result of trauma, caries, previous restorations and endodontic procedures, reducing the fracture resistance of the tooth. Endodontic access in combination with the earlier loss of one or both marginal ridges leave the tooth at serious risk of fracture, even if it was reduced out of direct occlusal contact before endodontic treatment began. There is convincing evidence that cuspal coverage after root canal treatment should be provided for posterior teeth. Access preparations result in greater cuspal flexure, increasing the probability of cuspal fracture. The presence of cuspal coverage is the only significant restorative variable to predict long-term success for such teeth. This conclusion is based on an independent, retrospective study of 608 endodontically treated teeth that evaluated the factors that affected survival during a 10-years period $^{(7)}$. Although there are several studies on ETT, treatment planning and the choice of material for the restoration are still controversial, and some criteria must particularly be considered. The remaining coronal tooth structure and functional requirements are important factors to be considered in deciding the treatment planning ${ }^{(8)}$.

Posterior teeth exhibit different restorative needs due to occlusal forces placed on them during function. Many attempts have been made to replace conventional full coverage restoration with various restorative materials. During the last 30 years, evolution of adhesive philosophy in dentistry and the high bonding performance achieved by modern adhesive systems have gradually changed the dogma that devitalized tooth should be restored with post, core and crown. Adhesion ensures sufficient material retention without the need of aggressive macro-retentive technique ${ }^{(9)}$.

The hypothesis of this study was that there will be a difference in fracture resistance between the type of restoration used and the material of construction.

\section{MATERIAL AND METHODS}

\section{Grouping:}

A total of twenty four specimens were divided equally into two main groups according to the type of restoration (12 endocrowns and 12 onlays). Each group was further subdivided equally into two subgroups $(n=6)$ according to the type of ceramic material used (IPS Emax Cad (Ivoclar Vivadent, Liechtenstein, VITA Enamic, Vita Zahnfabrik, Germany).

\section{Preparation of specimens:}

Twenty-four freshly extracted sound human mandibular first molar teeth were collected for use in the study. An ultrasonic scaler (Woodpecker UDS-K LED) was used to remove calculus deposits, debris and soft tissue remnants from these teeth. Teeth were then disinfected in concentrated sodium hypochlorite solution for 10 minutes. All 
teeth were examined under $4 \mathrm{x}$ magnification loops (HEINE Optotechnik GmbH \& Co.KG) to detect any cracks, caries or old restorations. Defective teeth were excluded from the study and replaced with sound teeth. Teeth were cleaned and stored in $0.2 \%$ thymol solution ${ }^{(10)}$ at room temperature until the time of testing.

\section{Endodontic procedures:}

Access cavity preparation was done for deroofing of pulp chamber of each tooth. The working length was determined by inserting a \#10 size K-file (Dentsply Tulsa Dental Specialties) into the canal and determining the point at which the file exited the apical foramen of the root. The file length was then reduced by $0.5 \mathrm{~mm}$ and the glide path was established. Root canal preparation (cleaning and shaping) was performed using machine-driven rotary files (ProTaper) with following the sequence: S1, S2, F1, F2, F3 (Dentsply Maillefer, Ballaigues, Switzerland). Sodium hypochlorite solution (2.5\%) was used to irrigate the canals throughout the instrumentation procedure. The canals were dried with paper points and obturated with matching Gutta-Percha points (Roeko, Langenau, Germany) using cold lateral condensation technique and ADSEAL sealer. A heated instrument was used to remove excess gutta percha.

\section{Periodontal membrane Simulation:}

The roots of each tooth was covered with a (0.3$0.5) \mathrm{mm}$-thick layer of polyvinyl acetate (EasyVac Gasket, $3 A$ MEDES) using a vacuum forming machine (Keystone Vac Former (AA103), USA) to simulate the physiological tooth movement.

\section{Mounting the abutments:}

A special machine-milled aluminum holder was designed and fabricated to mount abutment teeth inside acrylic blocks (Acrostone, Acrostone Dental Manufacture, Egypt). A parallometer (Paraflex, $B E G O$-Germany) was used to ensure accurate vertical centralization of the tooth in the holder.

\section{Model Adjustment:}

A Dentoform cast model (Elbanna model, Egypt) was used in this study for the use of its teeth as biogenaric copy during CAD/ CAM fabrication of specimens. The mandibular arch was replaced by an extra hard stone replica to facilitate cutting when needed. After the optical impression of teeth of the model was made, the cast model was adjusted to accommodate the size of the block by making a space at the site of mandibular first molar sockets. This space allows lateral insertion and removal of the acrylic block to the model. Now we have a stone mandibular arch in which the first molar was replaced by acrylic block containing natural molar (Figure 9). Soft wax was applied around each block in the model socket to prevent movements of the block. A custom-made index was constructed to standardize the occlusal clearance for all teeth and was used during placement of each block.

\section{Abutment preparation:}

Preparation of all abutments was completed using a milling machine to ensure a standardized tooth preparation.

\section{Endocrown preparation:}

The teeth were prepared by the same researcher to receive the endocrown restorations with occlusal coverage and pulp chamber extensions. Accordingly, the occlusal surfaces were horizontally reduced by $1.5 \mathrm{~mm}$. In addition to the occlusal preparation, $2 \mathrm{~mm}$ extra vertical reduction was made on the buccal surface occlusally. The internal walls of the pulp chamber were smoothed in accordance with the path of insertion. A standardized cavity preparation was performed in all teeth limited to removal of undercut areas of the pulp chamber and alignment of its axial walls with an internal taper of 8-10 degrees using a diamond-coated tapered stainless-steel bur held perpendicular to the pulpal floor. All internal line angles were rounded and smoothed using the same type of bur. Axial walls were prepared from the pulpal side to provide for 
a standardized cavity wall thickness of $3.0 \pm 0.2$ $\mathrm{mm}$ measured with a digital caliber.

\section{Onlay preparation}

The pulp chambers were firstly filled with composite resin, then, an MOD class 2 cavity was prepared. All preparations were completed along the longitudinal axis of the teeth. The pulpal floor depths were prepared to depths of $2.5 \mathrm{~mm}$ from the occlusal Cavo-surface margin of the preparations. A reduction of $2 \mathrm{~mm}$ on the buccal (functional) cusps and $1.5 \mathrm{~mm}$ on the lingual (non-functional) cusps was established. The width of the gingival floor preparations was $1 \mathrm{~mm}$. The width of the occlusal isthmus was $1 / 3^{\text {rd }}$ the width of the inter-cuspal width. Proximal preparations were finished $1 \mathrm{~mm}$ occlusal to the cemento-enamel junction. Internal line angles were rounded to smooth the preparations.

\section{Fabrication of the restoration:}

All Restorations were fabricated using the Cerec in Lab system (Sirona, Dentsply, Germany) which consists of personal computer, inEosX5 Blue scanner, and inLab MC X5 milling unit. The restoration design was accomplished by Cerec inLab (inLab Software 18.0).

\section{Surface treatment of the restorations:}

For IPS Emax: The intaglio of each restoration was etched with DentoBond Porcelain Etch (hydrofluoric acid $8 \%$ ) for 20 seconds $^{(11)}$, then rinsed with water and dried with moisture-free oil-free air until a frosted white appearance was evident. Then, the DentoBond Porcelain Silane (DentoBond Porcelain Etch, ITENA, France) was brushed on the etched ceramic surface and left for 1 minute, then dried well with moisture free oil-free compressed air according to the manufacturer's instructions.

For VITA ENAMIC: The intaglio of the restoration was etched with DentoBond Porcelain Etch (hydrofluoric acid 8\%) for 60 seconds, then rinsed with water and dried with moisture-free oilfree air until a frosted white appearance was evident. After that, restorations were cleaned using $70 \%$ ethyl alcohol in an ultrasonic cleaner. DentoBond Porcelain Silane was then brushed on and left for 1 minute then dried well with moisture-free oil-free compressed air.

\section{Surface treatment of abutments:}

Teeth were washed with water and dried with air taking care not to desiccate the tooth surface. Prepared teeth were selectively etched with Scotchbond (3MESPE) Etchant for $30 \mathrm{secs}$ for enamel only according to the manufacturer's instructions, then washed thoroughly with an airwater stream for 15 seconds, and air-dried.

\section{Application of the cement:}

The mixing tip was attached to the tube of TotalCem (Itena, France) resin cement syringe. The material was dispensed directly onto the restoration

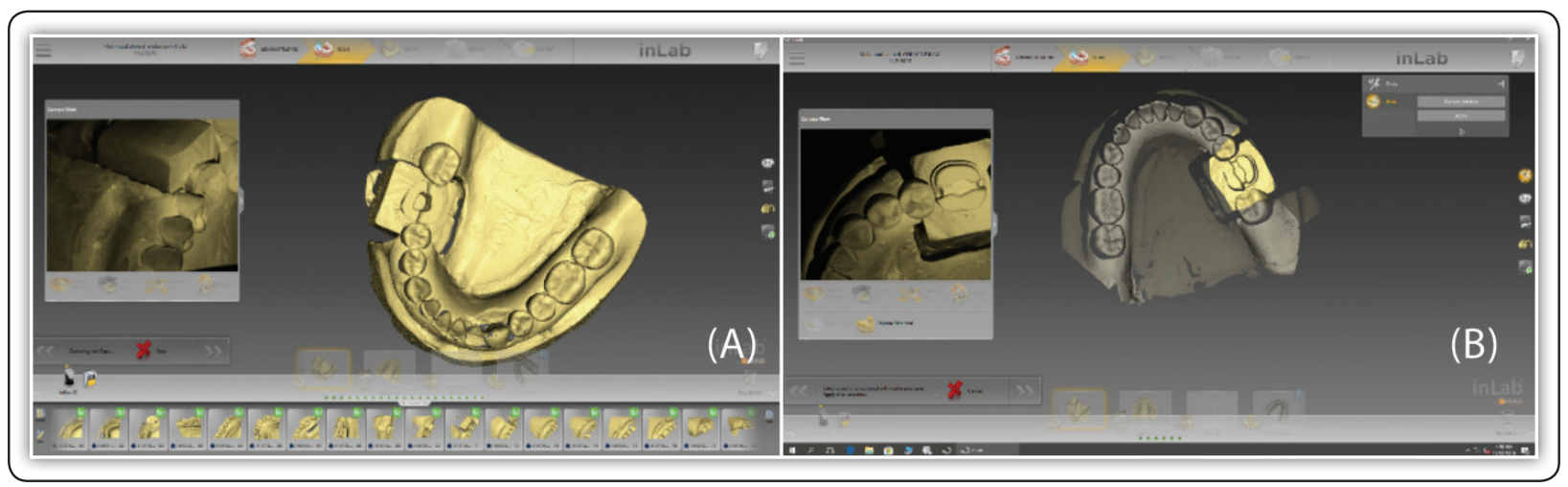

FIG (1) Scanning of the tooth sample; (A) Endocrown, (B) onlay. 
covering all surfaces. Then the restoration was seated gently on the preparation allowing the cement to flow from all sides. A custom-made device was used to standardize the applied force during cementation having a $5 \mathrm{~kg}$ weight. When the restoration was properly seated, the material was allowed to reach the gel state by letting it to self-cure for 90 seconds. Then tack curing for 2 seconds/surface according to the manufacturer instructions was done. All excess cement was then removed from all surfaces with a hand scaler. After removal of excess cements, the luting material was finally cured using a light-curing unit (700 Mw/Cm2. Elipar 2500) For 100 seconds (20 seconds/surface).

\section{Test procedure:}

All specimens were individually mounted on a computer-controlled material testing machine (Model 3345; Instron Industrial Products, Norwood, MA, USA) with a load cell of $5 \mathrm{kN}$, data were recorded using computer software (Instron ${ }^{\circledR}$ Bluehill Lite Software). Specimens were secured to the lower fixed compartment of testing machine by tightening screws. Fracture test was done by compressive mode of load applied occlusally using a metallic rod with round tip (5.6 $\mathrm{mm}$ diameter) attached to the upper movable compartment of testing machine traveling at a cross-head speed of $1 \mathrm{~mm} / \mathrm{min}$ with tin foil sheet in-between to achieve homogenous stress distribution and minimization of the transmission of local force peaks. The load at failure manifested by an audible crack and confirmed by a sharp drop at load-deflection curve recorded using computer software (Bluehill Lite Software Instron ${ }^{\circledR}$ Instruments). The load required to fracture was recorded in Newton. After the fracture resistance test was done, the fractured specimens were collected, and the mode of fracture was visually examined and classified according to the following patterns: favorable fracture (repairable fracture of the teeth/restorations above the level of bone simulation), and unfavorable fracture (Irreparable fractures below the level of bone simulation).
Data were represented by mean, standard deviation (SD), median (M), with 95\% Confidence Interval $(95 \% \mathrm{CI})$ values. Two-way ANOVA test post hoc Tuckey tests and descriptive statistics were used to compare between different designs and materials. The significance level was set to $\mathrm{P} \leq 0,05$. Statistical analysis was performed with IBM ${ }^{\circledR} *$ SPSS ${ }^{\circledR}{ }^{\circledR}$ Statistics Version 20 at $95 \%$ confidence interval.

\section{RESULTS}

The results of this study showed that generally Endocrown restorations had higher fracture resistance values than onlays. Also ceramic Emax materials had higher fracture values than the hybrid ceramic and was statistically significant. All values are represented in table (1).

TABLE (1) Comparison between different restorations of different materials.

\begin{tabular}{|c|c|c|c|c|}
\hline \multicolumn{2}{|c|}{ Emax } & Mean (N) & SD (N) & P \\
\cline { 2 - 4 } & Endocrown & 3080.24 & 883.60 & \multirow{2}{*}{$0.30^{\mathrm{ns}}$} \\
\hline \multirow{3}{*}{ V.Enamic } & Endocrown & 1976.16 & 339.66 & \multirow{2}{*}{$0.95^{\mathrm{ns}}$} \\
\cline { 2 - 4 } & Onlay & 1995.58 & 743.49 & \\
\hline \multirow{3}{*}{ Endocrown } & Emax & 3080.24 & 883.60 & \multirow{2}{*}{$0.02^{\mathrm{s}}$} \\
\cline { 2 - 4 } & V.Enamic & 1976.16 & 339.66 & \\
\hline \multirow{3}{*}{ Onlay } & Emax & 2628.91 & 478.21 & \multirow{2}{*}{$0.11^{\mathrm{ns}}$} \\
\cline { 2 - 4 } & V.Enamic & 1995.58 & 743.49 & \\
\hline
\end{tabular}

\section{DISCUSSION}

Rehabilitation of the endodontically treated teeth with severe crown damage represents a clinical challenge. While making a decision on the treatment of endodontically treated teeth with extensive loss of coronal structure, it should be aimed at protecting and strengthening the remaining tooth structure ${ }^{(12)}$. Therefore, the materials available in the market and the prosthetic treatment choices play an important role in the longevity of both the restoration and devitalized teeth $^{(13)}$. 
The introduction of adhesive techniques has revolutionized the restoration of endodontically treated teeth, since it is no longer necessary to take the mechanical retention into account, but instead rely on micromechanical and molecular retention provided by the adhesive procedure. Bearing this in mind, the more area between the tooth and the restoration (interface area), the higher probability of survival of the restoration ${ }^{(14)}$. In a study made by Bitter et al., they reported that the restoration of cavities with remaining palatal and buccal wall using Onlay restorations with proximal boxes and cusp coverage is better than with Inlay restorations without cusp coverage ${ }^{(15)}$.

According to the results of this study, the research hypothesis was partially accepted. Although IPS Emax showed higher value of fracture resistance than Vita Enamic, all the tested restoration designs and indirect restorative materials showed higher values in fracture resistance of endodontically treated teeth than that of natural teeth ${ }^{(16)}$.

Results of the current study demonstrated that there were no statistically significant differences between endocrown and onlay within the two materials, where the mean and standard deviation (SD) of both endocrown and onlay groups were $(3080.24 \pm 883.60 \mathrm{~N}$ and $2628.91 \pm 478.21$ N) for Emax, and were $(1976.16 \pm 339.66 \mathrm{~N}$ and $1995.58 \pm 743.49 \mathrm{~N})$ for vita Enamic respectively. However, there was a statistically significant difference in endocrown restorations fabricated either from E-max or Vita Enamic respectively (3080.24 $\mathrm{N}$ and $1976.16 \mathrm{~N}$ ).

The high fracture resistance recorded by endocrowns fabricated from Emax could be attributed to the high mechanical properties of this material. The microstructure of the LD includes needle-like particles with different orientations. Its elastic modulus ( $\sim 64 \mathrm{GPa})$ and particle size (from 0.5 to $4 \mu \mathrm{m}$ ). The higher crystalline content (approximately $70 \%$ by volume) and densely packed crystalline structure of LD in addition to an elongate grain structure is well suited to providing a respectable toughness by inhibiting crack propagation and increase the mechanical strength. Even if cracks were to form, they would become trapped within the crystals, potentially preventing further propagation ${ }^{(17)}$.

In this study, the lowest fracture strength mean value was recorded by endocrowns fabricated from hybrid ceramic material VITA ENAMIC (1976.16 $\mathrm{N})$. This could be attributed to the relatively low mechanical properties of this material including low flexural strength (150-160 MPa) and low fracture toughness $\left(1.5 \mathrm{MPa} \mathrm{m}{ }^{1 / 2}\right)$. Another possible factor may be the hybrid nature of this material as it is composed of interconnected networks of ceramic and polymer, which leads to different rates of ablation for ceramic and polymer during the grinding and polishing processes, that may result in microcracks in the network boundaries, and this is assumed to decrease the mechanical properties of the material ${ }^{(18)}$. Moreover, in a hybrid material, failure could be initiated from any weak point of the microstructure, like the polymer in a polymerinfiltrated ceramic ${ }^{(19)}$.

The results of the current study is in agreement with the findings of Bilkhair ${ }^{(20)}$ who compared the fracture strength of monolithic crowns fabricated from hybrid dental ceramic with those fabricated from lithium disilicate and feldspathic ceramics. They found that the fracture strength of crowns fabricated from hybrid dental ceramic was lower than that of lithium disilicate crowns. This finding is also in agreement with Sieper et $a l^{(21)}$ who compared the fracture strength of all-ceramic crowns fabricated from hybrid dental ceramic, lithium disilicate and zirconia-reinforced lithium silicate and found that the lowest fracture strength of all-ceramic crowns was recorded by crowns fabricated from hybrid dental ceramic.

It has been reported that several factors play an important role on the performance and longevity of ceramic restorations, such as the strength and 
thickness of the ceramic, the compatibility of the elastic moduli of the ceramics and teeth, and the adaptation of the restorations to the interfacial bonding surface ${ }^{(22)}$. Regarding the restoration type in case of Emax there was no statistically significant difference between endocrown and onlay restorations, although endocrowns have a higher fracture resistance $(3080.24 \mathrm{~N})$ than onlays $(2628.91 \mathrm{~N})$. Higher fracture strength values of endocrown groups may also be attributed to the thickness of the ceramic occlusal portion of endocrown. In vitro studies have shown that the fracture resistance values of glass ceramic crowns increase with increasing occlusal thickness ${ }^{(23)}$. This assumption was verified by a study that reported that the fracture resistance of endocrowns with an occlusal thickness of $5.5 \mathrm{~mm}$ was two times higher than that of ceramic crowns with a classic preparation and an occlusal thickness of $1.5 \mathrm{~mm}^{(24)}$ . In case of Vita Enamic There was no statistically significant difference between endocrown and onlay as $\mathrm{p}=0.95$ Despite of Onlay has higher fracture resistance $(1995.58 \mathrm{~N})$ than endocrown $(1976.16 \mathrm{~N})$

The large standard deviations obtained in this study could be attributed to various factors including that; although the extracted teeth were carefully selected however it might contain subclinical flaws or irregularities and morphological variations. Also bonding to different teeth substrates in which they may have different composition and/or degree of calcification $^{(25)}$. However, these variations are likely to exist in clinical situations as well. Therefore, the range of values can be considered relevant as it was related to actual performance.

Most restorations showed a catastrophic mode of fracture, which includes combined fracture of the restorations and the tooth structure. This may be attributed to teeth were subjected to a compressive load until the fracture. Furthermore, high bond strength that occurred between ceramic restorations and tooth surface by resin cement may have played a role in this ${ }^{(26)}$.
It is worth mentioning that, this in-vitro study has limitations, and therefore the results should be interpreted with caution. In contrast to in-vitro testing, the clinical load capability of root-filled teeth is influenced by additional factors like the number of adjacent teeth, the number of occlusal contacts, tooth position in the dental arch and apical status (27). The method of loading might be controversial because the compressive static loading used in the present study is different from the dynamic loading in the mouth ${ }^{(28)}$.

\section{CONCLUSION}

Within the limits of this in vitro study, the following can be concluded:

- Endocrowns and onlays can be used safely in terms of fracture strength as both have values which exceed the physiologic requirements.

\section{REFERENCES}

1. Huang T-JG, Schilder H, Nathanson D. Effects of moisture content and endodontic treatment on some mechanical properties of human dentin. Journal of Endodontics. 1992;18(5):209-15.

2. Gulabivala K, Stock CJR, Gulabivala K, Walker RT. Root canal system preparation. Endodontics: Elsevier, Mosby, Edinburgh; New York; 2004. p. 142-4.

3. Trope M, Ray Jr HL. Resistance to fracture of endodontically treated roots. Oral surgery, oral medicine, oral pathology. 1992;73(1):99-102.

4. Reeh ES, Messer HH, Douglas WH. Reduction in tooth stiffness as a result of endodontic and restorative procedures. Journal of endodontics. 1989;15(11):512-6.

5. Cruz-Filho AM, Sousa-Neto MD, Saquy PC, Pécora JD. Evaluation of the effect of EDTAC, CDTA, and EGTA on radicular dentin microhardness. Journal of endodontics. 2001;27(3):183-4.

6. Wahab F. Restoring of endodontically treated tooth. Concepts and Techniques, The Saudi Dental Journal. 2004;16(2):6169.

7. Cheung GS, Chan TK. Long-term survival of primary root canal treatment carried out in a dental teaching hospital. International endodontic journal. 2003;36(2):117-28. 
8. Faria ACL, Rodrigues RCS, de Almeida Antunes RP, de Mattos MdGC, Ribeiro RF. Endodontically treated teeth: characteristics and considerations to restore them. Journal of prosthodontic research. 2011;55(2):69-74.

9. Krejci I, Duc O, Dietschi D, de Campos E. Marginal adaptation, retention and fracture resistance of adhesive composite restorations on devital teeth with and without posts. Operative Dentistry. 2003;28(2):127-35.

10. Soares CJ, Pizi ECG, Fonseca RB, Martins LRM. Influence of root embedment material and periodontal ligament simulation on fracture resistance tests. Brazilian Oral Research. 2005;19(1):11-6.

11. Straface A, Rupp L, Gintaute A, Fischer J, Zitzmann NU, Rohr N. HF etching of CAD/CAM materials: influence of HF concentration and etching time on shear bond strength. Head \& face medicine. 2019;15(1):21.

12. Linn J, Messer HH. Effect of restorative procedures on the strength of endodontically treated molars. Journal of Endodontics. 1994;20(10):479-85.

13. Saunders WP, Saunders EM. Coronal leakage as a cause of failure in root-canal therapy: a review. Dental Traumatology. 1994;10(3):105-8.

14. Cheung W. A review of the management of endodontically treated teeth: Post, core and the final restoration. The Journal of the American Dental Association. 2005;136(5):6119.

15. ElAyouti A, Serry MI, Geis-Gerstorfer J, Löst C. Influence of cusp coverage on the fracture resistance of premolars with endodontic access cavities. International Endodontic Journal. 2011;44(6):543-9.

16. Carvalho AO, Bruzi G, Giannini M, Magne P. Fatigue resistance of CAD/CAM complete crowns with a simplified cementation process. The Journal of prosthetic dentistry. 2014;111(4):310-7.

17. de Carvalho Ramos N, Campos TMB, de La Paz IS, Machado JPB, Bottino MA, Cesar PF, et al. Microstructure characterization and SCG of newly engineered dental ceramics. Dental Materials. 2016;32(7):870-8.

18. Della Bona A, Corazza PH, Zhang Y. Characterization of a polymer-infiltrated ceramic-network material. Dental Materials. 2014;30(5):564-9.
19. Homaei E, Farhangdoost K, Tsoi JKH, Matinlinna JP, Pow EHN. Static and fatigue mechanical behavior of three dental CAD/CAM ceramics. Journal of the mechanical behavior of biomedical materials. 2016;59:304-13.

20. Jassim ZM, Majeed MA. Comparative Evaluation of the Fracture Strength of Monolithic Crowns Fabricated from Different all-ceramic CAD/CAM Materials (an in vitro study). Biomedical and Pharmacology Journal. 2018;11(3):1689-97.

21. Sieper K, Wille S, Kern M. Fracture strength of lithium disilicate crowns compared to polymer-infiltrated ceramicnetwork and zirconia reinforced lithium silicate crowns. Journal of the mechanical behavior of biomedical materials. 2017;74:342-8.

22. Kelly JR. Clinically relevant approach to failure testing of all-ceramic restorations. The Journal of prosthetic dentistry. 1999;81(6):652-61

23. Tsai Y-L, Petsche PE, Anusavice KJ, Yang MC. Influence of glass-ceramic thickness on Hertzian and bulk fracture mechanisms. International Journal of Prosthodontics. 1998;11(1).

24. Mörmann WH, Bindl A, Lüthy H, Rathke A. Effects of preparation and luting system on all-ceramic computergenerated crowns. International Journal of Prosthodontics. 1998;11(4).

25. Zhang Y, Lee JJW, Srikanth R, Lawn BR. Edge chipping and flexural resistance of monolithic ceramics. Dental materials. 2013;29(12):1201-8.

26. Burke FJT. Maximising the fracture resistance of dentine-bonded all-ceramic crowns. Journal of dentistry. 1999;27(3):169-73

27. Siso Ş, Hürmüzlü F, Turgut M, Altundaşar E, Serper A, Er K. Fracture resistance of the buccal cusps of root filled maxillary premolar teeth restored with various techniques. International endodontic journal. 2007;40(3):161-8.

28. Yamada Y, Tsubota Y, Fukushima S. Effect of restoration method on fracture resistance of endodontically treated maxillary premolars. International Journal of Prosthodontics. $2004 ; 17(1)$ 